ARTICLE

\title{
Estimate of Photonuclear Reaction in a Medical Linear Accelerator Using a Water-Equivalent Phantom
}

\author{
Toshioh FUJIBUCHI ${ }^{1,2, *}$, Satoshi OBARA ${ }^{2}$, Hitoshi $\mathrm{SATO}^{2}$, Masaru NAKAJIMA ${ }^{3}$, Nozomi KITAMURA ${ }^{3}$, \\ Tomoharu SATO ${ }^{3}$, Hiroaki KUMADA ${ }^{1}$, Takeji SAKAE ${ }^{1}$ and Tatsuya FUJISAKI ${ }^{2}$ \\ ${ }^{1}$ Graduate School of Comprehensive Human Sciences, University of Tsukuba, Ibaraki, Japan \\ ${ }^{2}$ School of Health Sciences, Ibaraki Prefectural University of Health Sciences, Ibaraki, Japan \\ ${ }^{3}$ Department of Radiation Oncology, Cancer Institute Hospital, Japanese Foundation for Cancer Research, Tokyo, Japan
}

\begin{abstract}
In recent radiotherapy techniques, neutrons are generated when the incident photon energy is higher than the threshold of $(\gamma, \mathrm{n})$ reaction. Reactions occur in the various target materials, as well as in the flattening filters and collimators comprising the head structure of the electron linac. The resulting secondary radiation may lead to secondary cancer in patients because of the increased radiation dose. We measured the secondary neutrons generated in the linac head, used for radiation therapy at 10 and $15 \mathrm{MV}$ using a water-equivalent phantom, gold foil, and solid state track detectors. We calculated the neutron distribution in a model of the same situation using the PHITS code. In Monte Carlo calculations, the scattering of thermal neutrons in the water phantom was confirmed. The contribution from these neutrons was $0.1 \%$ or less of that from X-rays at $10 \mathrm{MV}$. Because the cross-section of the photonuclear reaction increased in the high-energy linac, further examination is required. For $15 \mathrm{MV}$ X-rays, the amount of neutrons was 10 times higher than for $10 \mathrm{MV}$ X-rays. The PHITS code was created to simulate a photonuclear reaction and is therefore suitable for calculations involving these reactions in radioactive materials.
\end{abstract}

KEYWORDS: photonuclear reaction, medical linear accelerator, Monte Carlo, radiotherapy

\section{Introduction}

In radiation therapy facilities using medical electron linear accelerators, neutrons are generated by photonuclear reactions occurring between X-rays and the atomic nuclei in various materials, depending on the accelerator energy. ${ }^{1,2)}$ The relative biological effectiveness of a neutron varies according to its energy, and there is a risk of tissue damage and secondary cancer in patients subjected to neutron exposure. ${ }^{3)}$ The treatment of pediatric tumors has come under critical evaluation, because younger patients have a longer life expectancy. ${ }^{4-6)}$ In addition, secondary radiation may lead to radioactivation in the treatment room. Therefore, the evaluation of neutron dose is important in the medical field.

This research employed Monte Carlo calculations using a detailed structural model of the linac head. The simulated neutron distribution and energy spectrum of the linac apparatus represented radiation therapy using 10 and $15 \mathrm{MV}$ X-rays. MV stands for Mega-volt. It is a unit of electric potential. Multiple detectors, including an activation detector and CR-39 in a water-equivalent phantom were used to measure the patient body distribution and the radiation dose from secondary neutrons generated in the water-equivalent phantom.

\section{Material and Methods}

\section{Experimental Configuration}

The experiment employed a linac as follows: EXL-15SP
(Mitsubishi Electric) for 10-MV and CLINAC iX (Varian Medical Systems) for 15-MV X-rays. A tough water phantom (Kyoto Kagaku) was used as a water-equivalent phantom. Given that the source-surface distance (SSD) was $90 \mathrm{~cm}$, four $5 \mathrm{~cm}$-thick, $30 \mathrm{~cm} \times 30 \mathrm{~cm}$ phantoms (for a total thickness of $20 \mathrm{~cm}$ ) were arranged to simulate a human body.

\section{Monte Carlo Simulation}

PHITS (Particle and Heavy Ion Transport code System) Monte Carlo code (ver. 2.24) was used. ${ }^{7)}$ LA150 was used as nuclear data library. ${ }^{8)}$ It was necessary to reproduce the entire head in detail to simulate neutron generation and behavior. The target, a primary collimator, a mirror, a monitor dosimeter, a secondary collimator, a bending magnet, and a shielding block around the head were simulated for each linac. The simulated geometry of the Clinac $\mathrm{iX}$ is shown in Fig. 1. The field size was a fixed $10 \mathrm{~cm} \times 10 \mathrm{~cm}$ at the isocenter. 10 and $15 \mathrm{MeV}$ electron beams, irradiating the targets of each linac, were simulated. $\mathrm{MeV}$ stands for million electron volts. It is a unit of energy. The energy cut-offs were $0.01 \mathrm{MeV}$ for photons and electrons, and $1.0 \times 10^{-11} \mathrm{MeV}$ for neutrons. A dump file was placed behind the secondary collimator. The irradiation reaching the water-equivalent phantom was calculated based on this file. The energy spectrum and dose distribution of the photons, electrons, and neutrons in the water-equivalent phantom and around the head were evaluated.

*Corresponding author, E-mail: fujibuchi@ipu.ac.jp

(C) 2011 Atomic Energy Society of Japan, All Rights Reserved. 


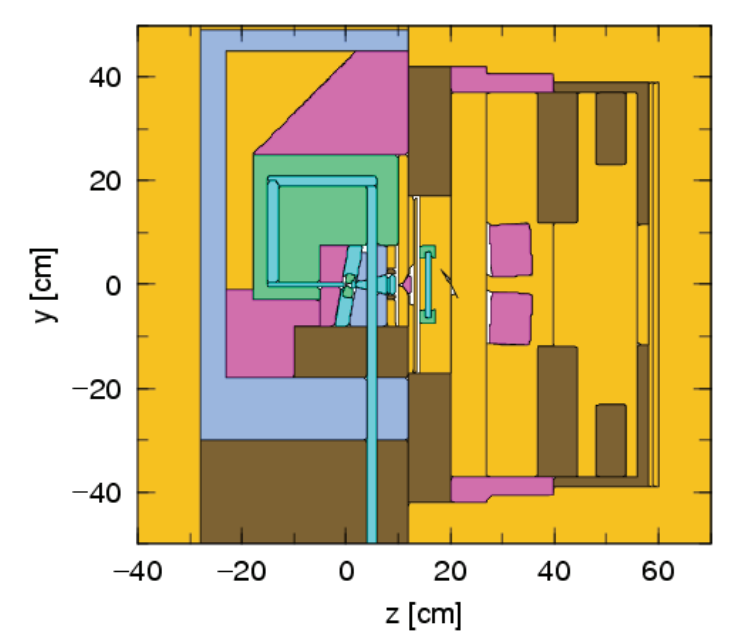

Fig. 1 Simulated geometry of the linac head and water equivalent phantom.

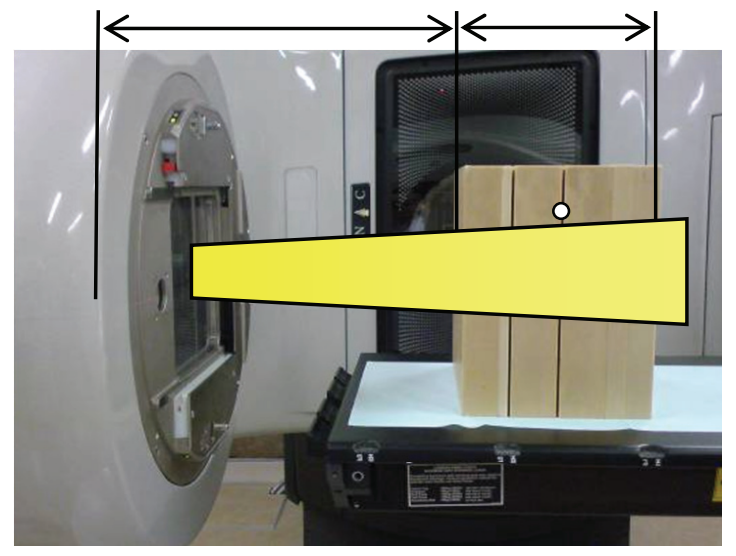

Fig. 2 Experimental setup of linac and water equivalent phantom. Glass dosimeters and CR-39s were positioned at the marked points.

\section{Dose Measurement of Photons and Neutrons Using Glass Dosimeters and Solid State Track Detectors}

As shown in Fig. 2, glass dosimeters and solid state track detectors (Chiyoda Technol Co.) were placed at three locations within the phantom, at source-chamber distances (SCD) of 95 and $100 \mathrm{~cm}$ on the beam axis, and at a SCD of $100 \mathrm{~cm}$ with a $10 \mathrm{~cm}$ lateral displacement from the beam axis. Using a field size of $10 \mathrm{~cm} \times 10 \mathrm{~cm}$, the dosimeters and detectors were irradiated with 10 and 15 MV X-rays from a 90-degree gantry angle, and the photon and neutron doses were measured.

\section{Measurement of Neutron Distribution Using Gold Foil}

As shown in Fig. 3, gold foil activation detectors $(\varphi 10 \mathrm{~mm} \times 0.05 \mathrm{~mm}$ thick $)$ were arranged within the phantom_from the central beam axis to the direction of Gun-Target and the direction of Right-Left at the position of $\mathrm{SCD}=90 \mathrm{~cm}, 95 \mathrm{~cm}, 100 \mathrm{~cm}, 105 \mathrm{~cm}$, and $110 \mathrm{~cm}$, respectively at $5 \mathrm{~cm}$ intervals, both along the beam axis and in the lateral and vertical directions. Gold foils are commonly used

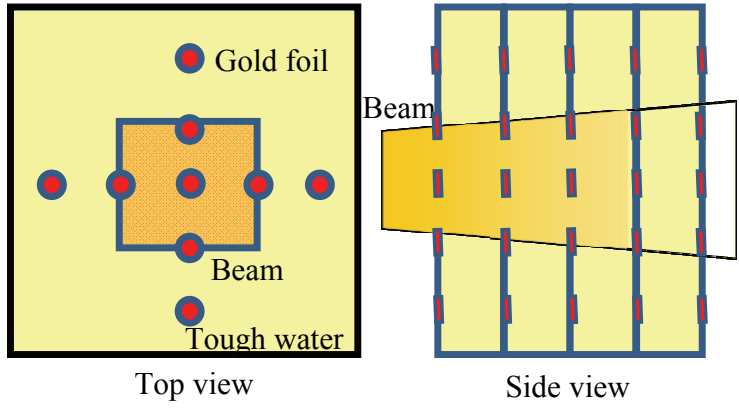

Fig. 3 Experimental setup of gold foils in the phantom.

to measure neutron fluence and energy spectrum, but the use of this many foils hampered the measurement of radioactivity. Therefore, the imaging plate (IP) was adapted for this measurement. The IP had a high sensitivity and a wide dynamic range for radioactive intensity, and provided two-dimensional information. Because the relative intensity of radioactivity was accurately obtained from many foil detectors simultaneously, half-life correction was unnecessary. Under a $10 \mathrm{~cm} \times 10 \mathrm{~cm}$ radiation field, the gold foils after radio-activation were attached to a BAS-MS (Fujifilm) to obtain images every 24 hours using an FLA-7000 (Fujifilm) $\mathrm{CR}$ readout device. The pixel values at the position of the gold foil on the image were measured as a relative radioactivity, and the changes over time were analyzed. One sample was measured to obtain the energy spectrum of the activated gold foil using a GMX-10190-P (SEIKO EG\&G) HP-Ge semiconductor detector and a MCA600 (Laboratory Equipment) pulse-height discriminator.

\section{Results}

\section{Monte Carlo Simulation}

The energy spectra of photons, electrons, and neutrons at the head entrance and in the water-equivalent phantom are shown in Fig. 4. The peak energy was $1 \mathrm{MeV}$. At $15 \mathrm{MV}$, the neutron dose was four orders of magnitude lower than that of the photon. At $10 \mathrm{MV}$, the neutron dose was five orders of magnitude lower than that of the photon. Fast neutron generation in the equipment was confirmed, and the serialization of these neutrons in the phantom was observed.

The photon depth doses along the central axis of 10 and 15 MV calculated by PHITS and measured by ion chamber are shown in Fig. 5. The dose distribution is normalized to the maximum dose. Measured and calculated values are similar. Photon and neutron profiles at $S C D=100 \mathrm{~cm}$ in a $15 \mathrm{MV}$ linac calculated by PHITS are shown in Fig. 6. The shapes of profiles were different between photon and neutron. The photon and neutron flux at $\mathrm{SCD}=95$ and $100 \mathrm{~cm}$, calculated using PHITS, are shown in Table 1. Ratios of the neutron to photon are about $10^{-3}$.

\section{Photon and Neutron Dose Measurement by Glass Do- simeter and Solid State Track Detectors}

The $1 \mathrm{~cm}$ dose equivalents of photons and neutrons at $\mathrm{SCD}=95$ and $100 \mathrm{~cm}$, as measured by glass dosimeter and CR-39, are shown in Table 2. The values were normalized so that the photon radiation dose was 100 monitor units 

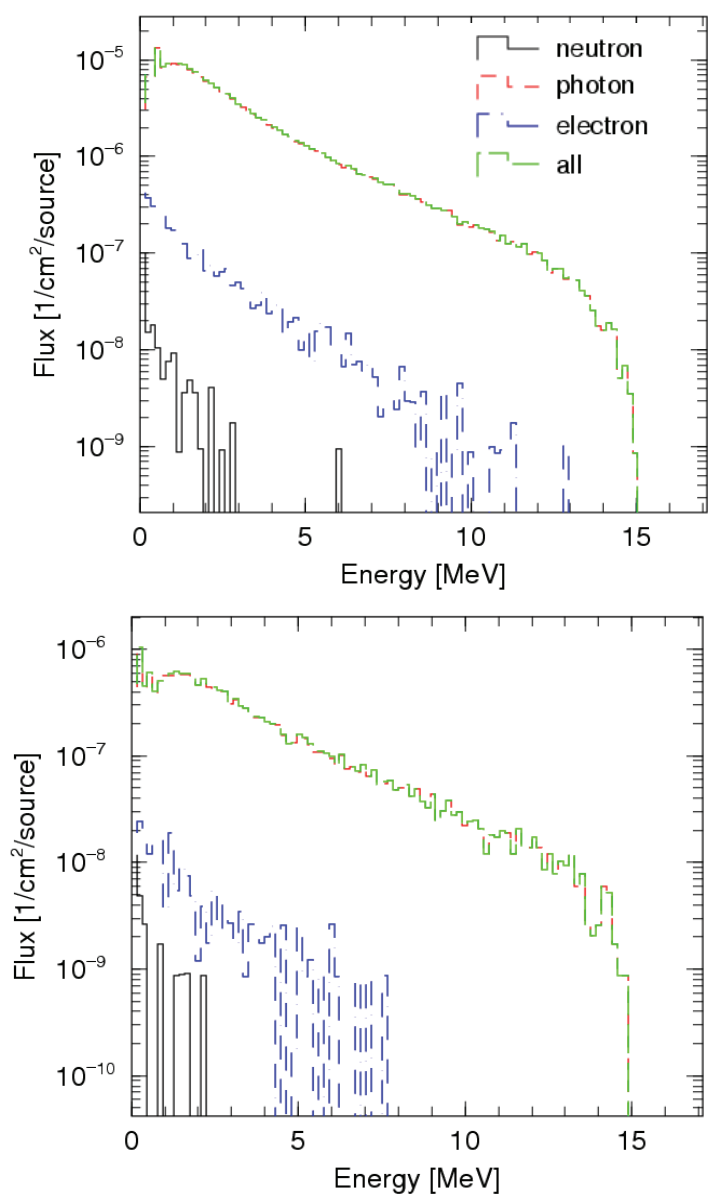

Fig. 4 Energy spectra of photons, electrons, and neutrons in the Clinac iX (15 MV), (a) at the linac head entrance, and (b) in the water-equivalent phantom.

(MU). The neutron dose was approximately three orders of magnitude smaller than the photon dose at $15 \mathrm{MV}$, and about four orders of magnitude smaller than the photon dose at $10 \mathrm{MV}$.

\section{Neutron Distribution Measured by Gold Foils}

A ${ }^{198} \mathrm{Au}$ energy peak $(411 \mathrm{keV})$ and ${ }^{196} \mathrm{Au}$ energy peaks $(335,354$, and $426 \mathrm{keV})$ were detected in the measurements of the activated gold foils using the HP-Ge semiconductor detector. Presumably, gold foils were activated with both the ${ }^{197} \mathrm{Au}(\mathrm{n}, \gamma){ }^{198} \mathrm{Au}$ reaction and the ${ }^{197} \mathrm{Au}(\gamma, \mathrm{n}){ }^{196} \mathrm{Au}$ reaction. When these nuclides are mixed, it is necessary to divide the PSL (photo-stimulated luminescence) intensity of the IP into ${ }^{198} \mathrm{Au}$ and ${ }^{196} \mathrm{Au}$ components. By adopting a least-squares method for the separation of PSL intensity of the IP over time, the ${ }^{198} \mathrm{Au}$ component was calculated.

The off-axis ratio of the neutron measured by gold foils and the off-axis ratio of the photon measured by the ionization chamber dosimeter in the $10 \mathrm{MV}$ linac are shown in Fig. 7. The neutron fluence had a smooth distribution, whereas the photon dose outside the radiation field is ten times lower than inside. The neutron distributions in the 10 and 15 MV linacs are shown in Fig. 8. There was no significant dependence of the profile on photon energy.

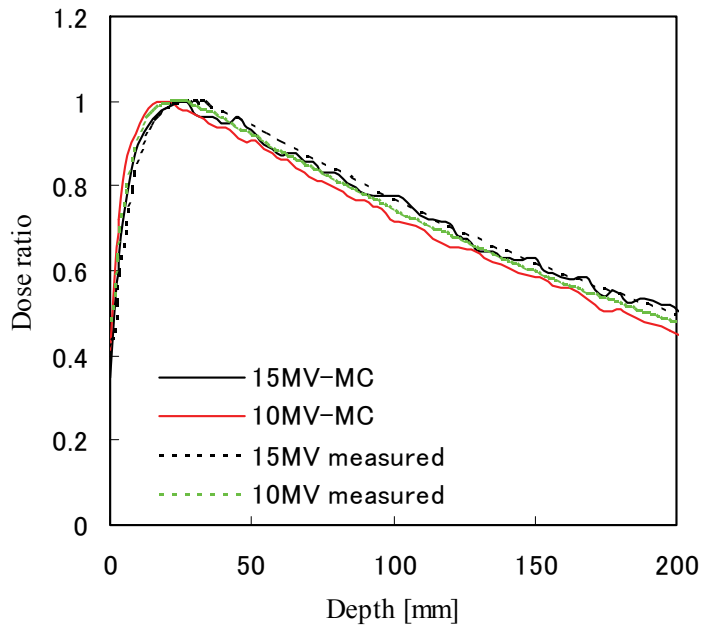

Fig. 5 Comparison between calculated and measured (PHITS) depth dose distribution from 10 and $15 \mathrm{MV}$. The dose distribu-tion is normalized to the maximum dose.

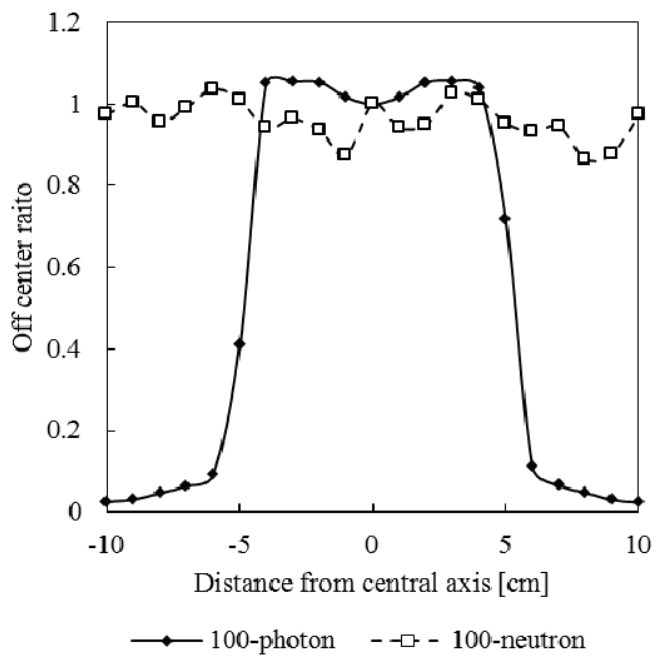

Fig. 6 Photon and neutron profiles at $\mathrm{SCD}=100 \mathrm{~cm}$ in a $15 \mathrm{MV}$ linac calculated by PHITS.

\section{Discussion}

The neutron to photon dose ratio was about $10^{-3}$ to $10^{-4}$ for standard irradiation with the electron linac. International Electrotechnical Commission (IEC) recommendations describe four leakage components: (1) downstream of adjustable beam limiting devices; (2) in the patient plane within a radius of $2 \mathrm{~m}$ (some states specify $1 \mathrm{~m}$ ); (3) inside the treatment room; and (4) outside the treatment room. The important factors to the exposure patient are the first two components. Version 2 of IEC 60601-2-1, the standard during the year 2006, recommends that the physical dose downstream of adjustable beam limiting devices due to leakage of primary particles be less than $2 \%$ of the prescribed dose. No recommendation is given for the neutron contamination component. Due to the growth of modulated x-ray therapy (IMXT) and the accompanying increase in monitor units and leakage radiation, a proposal for the upcoming 


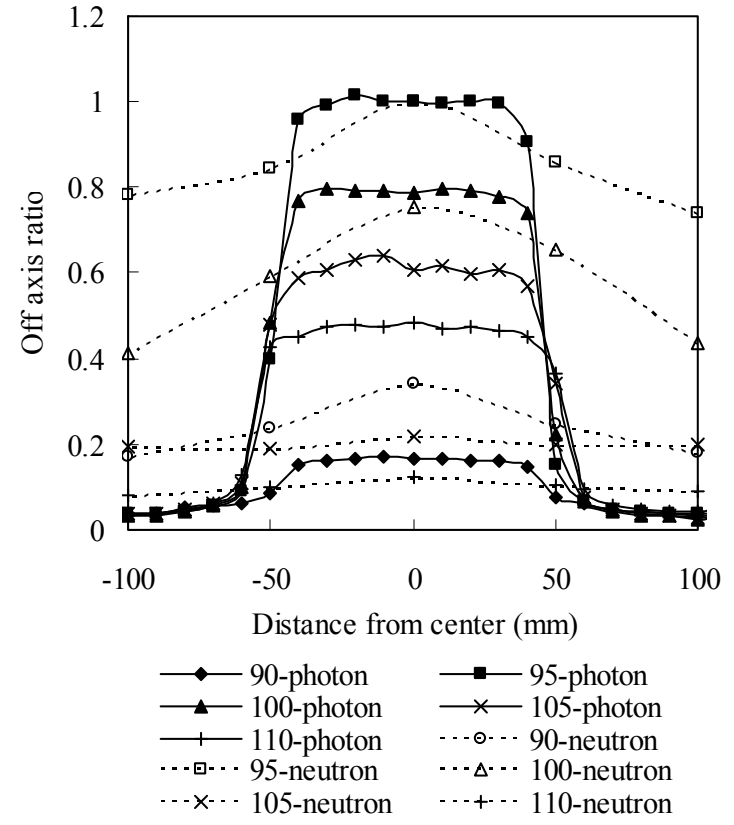

Fig. 7 Comparison of photon and neutron profiles at several phantom depths in a $15 \mathrm{MV}$ linac.

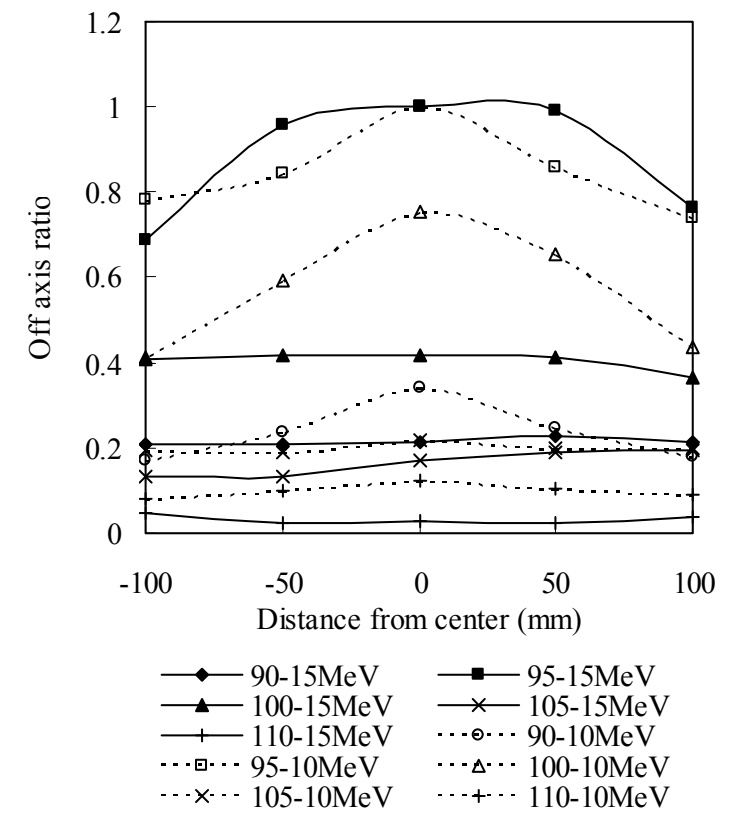

Fig. 8 Comparison of neutron profiles at several phantom depths in 10 and $15 \mathrm{MV}$ linacs.

version 3 of the IEC recommendations is that the average value behind adjustable beam limiting devices be limited to $0.75 \%$ while retaining the maximum value of $2 \%$. Outside of the projection of the beam limiting device, the existing IEC recommended standard is that the total physical dose from all ionizing radiation should be less than $0.1 \%$ of the prescribed dose. No quality factor (QF) or relative biological effectiveness (RBE) is considered but the physical dose from neutrons must be included as part of this $0.1 \%$. In the proposed revision, the non-neutron component is restricted to a maximum of $0.2 \%$ and an average of $0.1 \%$ while the neutron component is restricted to a maximum of $0.05 \%$ and an av-
Table 1 Calculated photon and neutron flux for 10 and $15 \mathrm{MV}$ $\mathrm{X}$-rays. These doses are normalized to neutron dose (15 MV, $\mathrm{SCD}=95 \mathrm{~cm}$ ) as 1 .

\begin{tabular}{lccccc}
\hline \multirow{2}{*}{$\begin{array}{c}\text { SCD } \\
(\mathrm{cm})\end{array} \begin{array}{c}\text { Distance } \\
\text { from beam } \\
\text { axis }(\mathrm{cm})\end{array}$} & \multicolumn{2}{c}{ Photon } & \multicolumn{2}{c}{ Neutron } \\
\cline { 3 - 6 } & 0 & 717 & 584 & 0.18 & 1 \\
\hline 95 & 0 & $15 \mathrm{MV}$ & $10 \mathrm{MV}$ & $15 \mathrm{MV}$ \\
100 & 0 & 678 & 593 & 0.07 & 0.86 \\
100 & 10 & 237 & 210 & 0.25 & 0.25 \\
\hline
\end{tabular}

Table 2 Photon and neutron doses under 10 and 15 MV X-ray irradiation. These doses are normalized to neutron dose $(15 \mathrm{MV}, \mathrm{SCD}=95 \mathrm{~cm})$ as 1 .

\begin{tabular}{|c|c|c|c|c|c|}
\hline \multirow{2}{*}{$\begin{array}{l}\mathrm{SCD} \\
(\mathrm{cm})\end{array}$} & \multirow{2}{*}{$\begin{array}{l}\text { Distance } \\
\text { from beam } \\
\text { axis }(\mathrm{cm})\end{array}$} & \multicolumn{2}{|c|}{$\begin{array}{c}\text { Photon } \\
\text { (glass dosimeter) }\end{array}$} & \multicolumn{2}{|c|}{$\begin{array}{l}\text { Neutron } \\
\text { (CR-39) }\end{array}$} \\
\hline & & $10 \mathrm{MV}$ & $15 \mathrm{MV}$ & $10 \mathrm{MV}$ & $15 \mathrm{MV}$ \\
\hline 95 & 0 & 1526 & 1340 & 0.18 & 1 \\
\hline 100 & 0 & 1324 & 1100 & 0.06 & 0.30 \\
\hline 100 & 10 & 0027 & 19 & 0.05 & 0.20 \\
\hline
\end{tabular}

erage of $0.02 \%$. The maximum of leakage ray was confirmed to be close to the value in $15 \mathrm{MV}$.

In the neutron measurements, there is a possibility of disarranging the place of neutron source by the detector placement and making the measurement difficult. Measuring the high energy photon and neutron energy spectra is difficult. Monte Carlo simulation can calculate energy spectrum with ease. The PHITS code was designed to simulate photonuclear reactions, and can be used to estimate the reactions in radioactive materials. Because the probability of a photonuclear reaction is low, lengthy simulations were required. Therefore, it is preferable to use parallel computation techniques.

Neutron measurements based on gold foils have a high sensitivity, but are unfortunately also sensitive to photons. As photon usage is prevalent in radiation therapy, neutron measurement based on gold foil can therefore be problematic.

Iron has a photonuclear reaction with a photon threshold value of $12 \mathrm{MeV}$. Wedge filter used to arrange the shape of the dose distribution is made of steel and the use of filter expectedly increases generation of neutron. ${ }^{9)}$ IMXT, the use of which is becoming increasingly wide-spread, increases the probability of a direct collision between a photon and a multi leaf collimator (MLC). As tungsten MLC has a lower threshold value for photonuclear reaction than steel, neutrons should be more rapidly generated in facilities using IMXT. The neutron dose measurement under such conditions will be discussed in the future.

\section{Conclusion}

Neutron doses and distributions in a water-equivalent phantom were calculated by PHITS and measured using gold 
foils and solid state track detectors. For complex irradiation, such as with a high energy or in IMXT, it is necessary to carefully determine the neutron dose, even though few neutrons are generated under normal conditions.

\section{References}

1) E. J. Hall, "Intensity-modulated radiation therapy, protons, and the risk of second cancers," Int. J. Radiat. Oncol. Biol. Phys., 65, 1-7 (2006).

2) M. F. Moyers, E. R. Benton, A. Ghebremedhin et al. "Leakage and scatter radiation from double scattering based proton beamline," Med.Phys., 35, 128-144 (2008).

3) C. Omar, C. M. Chang-Ming, "Photonuclear dose calculation for high-energy photon beams from Siemens and Varian linacs," Med. Phys., 30, 1990-2000 (2003).

4) S. Bhatia, C. Sklar, "Second cancers in survivors of childhood cancer," Nat. Rev. Cancer, 2, 124-132 (2002).
5) M. Stovall, C. R. Blackwell, J. Cundiff et al., "Fetal dose from radiotherapy with photon beams: report of AAPM Radiation Therapy Committee Task Group No. 36," Med. Phys., 22, 63-82 (1995).

6) F. Errico, M. Luszik-Bhadra, R. Nath et al., "Depthdose-equivalent and effective energies of photoneutrons generated by 6-18 MV X-ray beams for radiotherapy," Health Phys., 80, 4-11 (2001).

7) H. Iwase, K. Niita, T. Nakamura "Development of a general-purpose particle and heavy ion transport Monte Carlo code," J. Nuc. Sci. Technol., 39, 1142-1151 (2002).

8) M. B. Chadwick et al., LA-UR-99-1222; Nucl. Sci. Eng., 131, 293 (1999).

9) G. Seyyed-Mostafa, M. Asghar, M. Ehsan, "The impact of automatic wedge fileter on photonutron and photon spectra of an 18-MV photon beam," Radiat. Prot. Dosim., 138, 123-128, (2010). 